\title{
Erratum to: Halotolerant, alkaliphilic urease-producing bacteria from different climate zones and their application for biocementation of sand
}

\author{
Viktor Stabnikov • Jian Chu • Volodymyr Ivanov • \\ Yishan Li
}

Published online: 7 February 2014

(C) Springer Science+Business Media Dordrecht 2014

Erratum to: World J Microbiol Biotechnol

DOI 10.1007/s11274-013-1309-1

The name of the second author has been printed incorrectly in the original publication. The correct version is Jian Chu.

The online version of the original article can be found under doi:10.1007/s11274-013-1309-1.

V. Stabnikov $(\square)$

Department of Biotechnology and Microbiology,

National University of Food Technologies,

68 Vladymyrskaya Str., Kiev 01601, Ukraine

e-mail: vstabnikov@yandex.ru

V. Stabnikov $\cdot$ J. Chu

School of Civil and Environmental Engineering,

Nanyang Technological University, Singapore, Singapore

J. Chu $\cdot$ V. Ivanov $\cdot$ Y. Li

Department of Civil, Construction and Environmental

Engineering, Iowa State University, Ames, IA, USA 